\title{
A model-based method for reducing the sound speed induced errors in multi-beam echo-sounder bathymetric measurements
}

\author{
Mirjam Snellen, Kerstin Siemes, Dick G. Simons \\ Delft University of Technology, Faculty of Aerospace Engineering, Acoustic Remote Sensing Group \\ Kluyverweg 1 \\ 2629 HS, Delft, the Netherlands
}

\begin{abstract}
We present a method for accurately estimating the bathymetry from multi-beam echo-sounder (MBES) travel-time measurements in environments with large variations in the water column sound speeds (both temporally and spatially). In this type of environments the water column sound speeds at the time of the MBES transmission are often not known, preventing a reliable conversion from the measured travel-times to bathymetry. In addition, accurate sound speed information is required for the MBES beamsteering process. Situations where information regarding the prevailing sound speeds is insufficient occur, for example, in estuaries where fresh river water mixes with seawater. The method fully exploits the redundancy in the MBES measurements obtained from the overlap of adjacent swathes. It searches for those water column sound speeds that result in a maximum agreement in the bathymetry along the overlapping swathes. The Gauss-Newton method is employed for the optimization. This method is very efficient, requiring a few iterations only and therefore very well suited for processing the large datasets as typically obtained by the MBES. From simulations it is shown that the method performs well and allows for an almost exact determination of the bathymetry and sound speeds. In principle, the method eliminates the need for sound speed measurements, as long as sufficient overlap exists.
\end{abstract}

\section{INTRODUCTION}

Employing multi-beam echo-sounder (MBES) systems has proven to be a cost-efficient technique to map the bathymetry of sea- and river-floors within limited time. In order to cover a large area of the seafloor at once, an MBES sends out an acoustic pulse along a wide swathe perpendicular to the sailing direction. Beamsteering at reception allows for determining both the angle and the corresponding (two-way) travel-time of the received signals. For each ping, water depths along the swathe can be derived from the combination of travel-time and angle, provided that the local sound speed in the water column is known [1].

When conducting MBES measurements, typically two sets of sound speed measurements are taken. The first set consists of the sound speeds at the MBES transducer position which are usually measured continuously using a sensor under the keel of the ship. These sound speeds are employed for the beamsteering process. The second set consists of sound speed profiles acquired using for example a ConductivityTemperature-Depth (CTD) device. These sound speed profiles are required for determining the sound propagation through the water column, needed for deriving the bathymetry from the measured travel-times [2], [3].

Due to algae growth and other types of contamination on the sensor, the quality of the sound speed measurements at the transducer position sometimes gets degraded, resulting in beamsteering in directions that differ from those aimed for and which are unknown. In addition, due to time constraints, only a few sound speed profile measurements are conducted during a survey. For environments where the water column sound speed profiles vary rapidly (both temporally and spatially) this can result in errors in the derived bathymetry.

Both above mentioned errors result in a bathymetry that shows smiley- or droopy-like effects. In these cases, the depths measured in the outer parts of swathes are under- or overestimated. This is most obvious when parts of the swathes overlap, since then the measured depths in the overlap regions are not consistent with each other.

Here, we present a method for eliminating these errors, without the need for additional information on the sound speed profile. To this end, use is made of the fact that the seafloor will in general not vary much during the survey (several hours). By requiring the derived bathymetry along overlapping regions to coincide, the prevailing water column sound speeds and thus the bathymetry can be estimated by inversion. In theory, this approach eliminates the need for sound speed measurements as long as sufficient overlap along adjacent swathes is available [4], [5].

As a first step for assessing the feasibility of the proposed method simulations, have been carried out. Section II provides a description of the approach towards eliminating sound speed induced errors as proposed in this contribution. Section III presents the results of applying this method to simulated data. Conclusions regarding the applicability of the method are presented in Section IV. 


\section{DESCRIPTION OF THE APPROACH}

A typical multi-beam echo-sounder (MBES) in operation emits acoustic pulses within an opening angle of about 120 150 degrees in the across-track direction. Beamforming is applied to determine the corresponding two-way travel-times for a selected number of arrival angles. From the combinations of two-way travel-time and angle, water depths along the swathe are determined. This is done either by assuming straight sound rays for shallow water environments or by raytracing in case the curvature of the sound rays can not be neglected.

Inaccurate knowledge about the water column sound speeds result in an erroneous bathymetry in two ways:

1. Errors in the beamsteering process. In case the actual sound deviates from the measured sound speed, the actual beamsteering angles differ from the beamsteering angles aimed for and are unknown.

2. Errors in the conversion from the angle and traveltime combinations to water depths along the swathe.

Both error types result smiley- or droopy-like effects.

Fig. 1 shows the geometry of a typical MBES survey. A series of tracks are sailed parallel to each other. Track distances are such that a certain overlap exists between adjacent swathes.

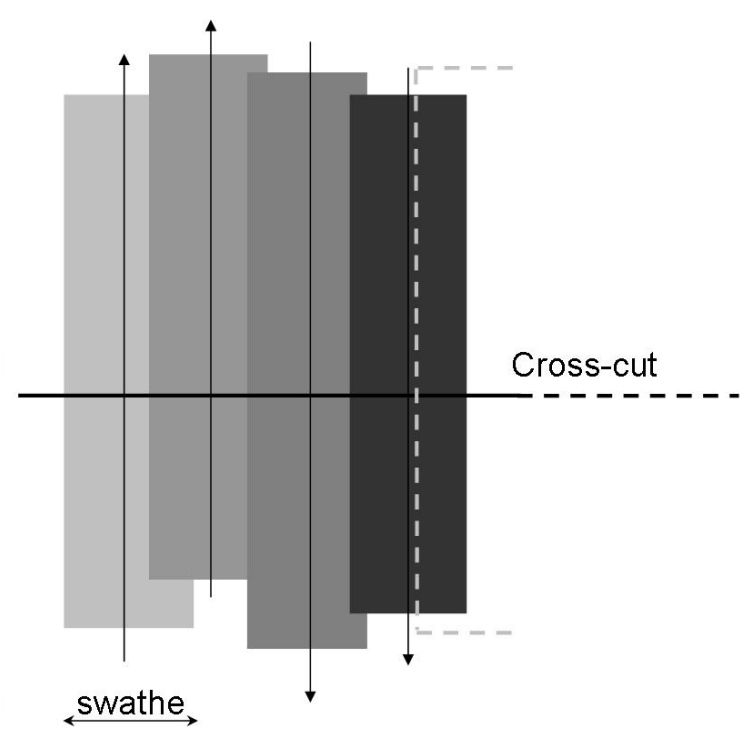

Figure 1. Typical MBES survey geometry. The arrows indicate the sailing direction. The rectangles indicate the area as measured per track.

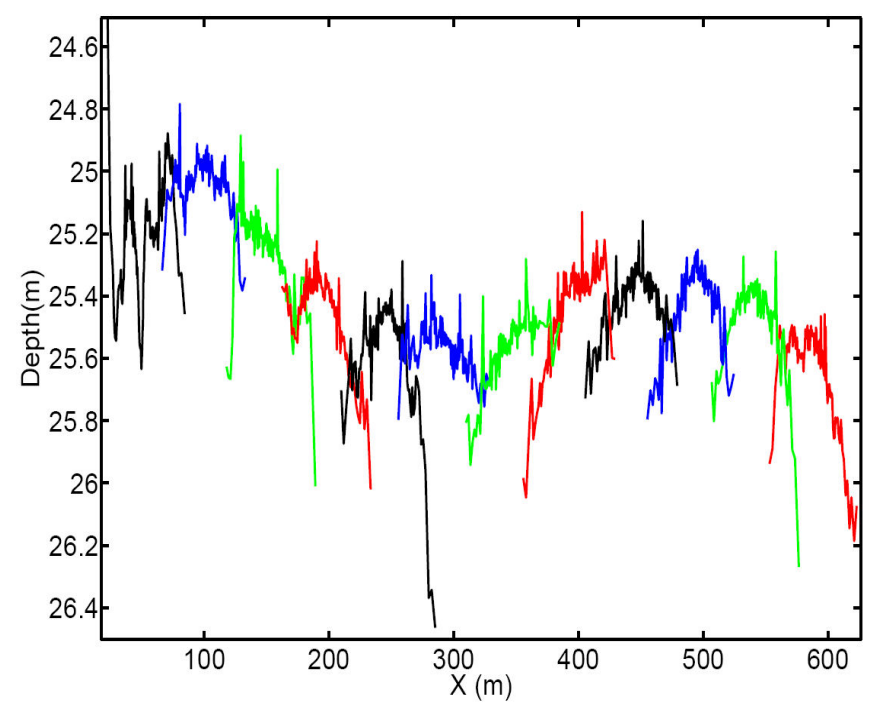

Figure 2. Example of 'droopy' effects. The MBES measurements were carried out near the entrance to the harbor of Rotterdam.

Fig. 2 shows an example of droopy-effects. The area in which these MBES were taken is located close to the entrance of the Rotterdam harbor, where mixing of fresh and salt water occurs.

The geometry of the MBES survey that resulted in the water depths as shown in Fig. 2 corresponds to that shown in Fig. 1. The number of parallel tracks amounted to 12 . The bathymetry was estimated from the measured travel-times, employing all sound speed information available, i.e., sound speeds measured at the transducer head for the beamsteering and a single sound speed profile for calculating the sound propagation through the water column. The colors indicate the bathymetry as estimated for each of the tracks. The droopy effects are clearly visible, resulting in differences in water depths along the overlapping parts of adjacent swathes of up to $0.5 \mathrm{~m}$.

\section{A. Energy Function}

In order to estimate the true bathymetry, we exploit the redundancy of measurements in the overlap region between two adjacent swathes. If we assume that the seafloor does not change during a survey, the depth measurements in the overlap regions should be the same.

The measurements are the two-way travel-times. We aim to minimize the function:

$E=\sum_{k=1}^{S} \sum_{j=1}^{N}\left(t_{k, j}-T_{k, j}\right)^{2}$ 
where $N$ and $S$ are the total numbers of MBES beams and swathes, respectively. The modeled two-way travel-times are denoted by $t_{k, j}$ and the measured two-way travel-times are denoted by $T_{k, j}$. The model that calculates $\mathrm{t}_{k, j}$, accounts for both the effect of sound speed on the beamsteering and on the propagation through the water column. The unknowns are the sound speed profiles for each of the swathes and the bathymetry. These unknowns should be selected such that $E$ becomes minimal.

Hereto the seafloor is modeled with an interpolated grid function. The water depths at the grid positions are the unknowns that are obtained through the minimization of $E$. Assuming a shallow water situation, we approximate the sound speed profile by a constant sound speed. This results in one unknown sound speed for each of the tracks. The unknowns to be optimized are thus the depths at the grid positions and the sound speeds for each of the swathes.

\section{B. Optimization method}

For minimizing $E$ use is made of the Gauss-Newton method. The optimization procedure is as follows.

For the seafloor, we use a fixed grid of horizontal positions, denoted $X_{n}$ in the across-track direction. At every position $X_{n}$, $Z\left(X_{n}\right)$ denotes the corresponding water depth. Between the grid points the depth is interpolated linearly. For every beam $j$ at angle $\theta_{k, j}$, the point where the acoustic beam impinges on the model seafloor is denoted as $\left(x_{k, j}, z_{k, j}\right)$. Fig. 3 shows a schematic overview of this model.

Mathematically, the function for $t_{k, j}$ can be derived by calculating the intersection between two lines: the sound ray and the line between the grid points in Fig. 3. The MBES is located at $\left(X_{k, M B E S}, Z_{k, M B E S}\right)$.

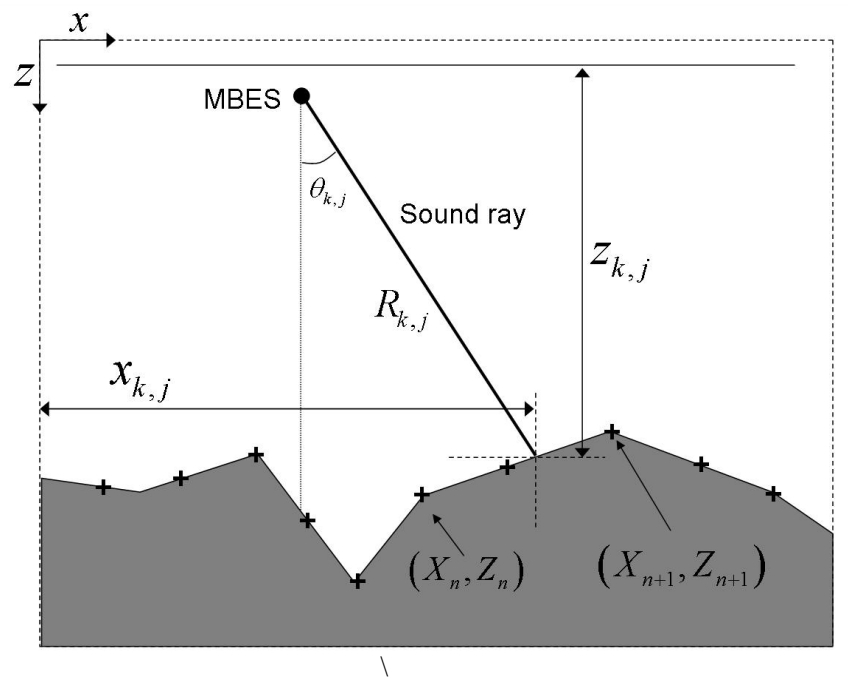

Figure 3. Schematic overview of the MBES measurements.
The position at which these tow lines intersect is given by:

$x_{k, j}=\frac{\frac{X_{k, M B E S}}{\tan \theta_{k, j}}+Z\left(X_{n}\right)-Z_{k, M B E S}-X_{n} \frac{Z\left(X_{n+1}\right)-Z\left(X_{n}\right)}{X_{n+1}-X_{n}}}{\frac{1}{\tan \theta_{k, j}}-\frac{Z\left(X_{n+1}\right)-Z\left(X_{n}\right)}{X_{n+1}-X_{n}}}$

and

$z_{k, j}=\frac{x_{k, j}-X_{k, M B E S}}{\tan \theta_{k, j}}+Z_{k, M B E S}$

Values for $t_{k, j}$ are calculated employing the water column sound speed $c k$ as

$t_{k, j}=\frac{2\left(x_{k, j}-X_{k, M B E S}\right)}{c_{k} \sin \theta_{k, j}}$

where $c k$ is the sound speed in swathe $k$.

We can write

$\mathbf{y}=A(\mathbf{x})$

with $\mathbf{y}$ the vector containing the measured travel-times, and $\mathbf{x}$ the vector containing all unknowns.

Solving for $\mathbf{x}$ in a least-squares sense requires the iterative Gauss-Newton approach. Hereto the expressions for the derivatives of $A$ to all unknowns have been determined.

\section{RESULTS}

To assess the applicability and performance of the abovedescribed method, it has been applied to simulated travel-time data. Hereto, a MBES measurement configuration is considered that is in agreement with practice.

As a first step, a situation where three parallel tracks are sailed is considered. The tracks are sailed $80 \mathrm{~m}$ apart. The MBES steering angles range from $-65^{\circ}$ to $+65^{\circ}$. The water depths are centered around $30 \mathrm{~m}$. The overlap along the swathes amounts to $40 \mathrm{~m}$.

The upper plot of Fig. 4 represents a situation where the water depths as derived from the angle/travel-time 
combinations show significant differences along the overlapping parts of the swathes, due to the use of erroneous sound speeds. The vertical dashed lines indicate the positions of the MBES for the three swathes. The true bathymetry is indicated as the grey surface. The water depths estimated for the different swathes are shown as colored lines. The true sound speed values for this situation amount to 1504, 1501, and $1498 \mathrm{~m} / \mathrm{s}$ for the different swathes. The sound speeds used for estimating the bathymetry, i.e., representing the "measured" sound speeds, are 1518, 1504, and $1510 \mathrm{~m} / \mathrm{s}$, respectively.

Application of the method described in this contribution allows for determining the true bathymetry, without the need for additional sound speed information. This is illustrated in the lower plot of Fig. 4, where the optimized bathymetry is shown by the black dots. These indicate the $Z\left(X_{n}\right)$ values. The grid points $X_{n}$ are separated by a 3-m distance.

Fig. 5 shows the sound speed estimates as a function of the number of Gauss-Newton iterations as black squares. The true sound speed is indicated by the dashed line. For all sound speeds a starting value of $1490 \mathrm{~m} / \mathrm{s}$ has been selected for the first Gauss-Newton iteration step. From the figure it becomes clear that a single iteration is already sufficient for obtaining the correct sound speed values. The number of unknowns amounts to 97 , i.e., 3 sound speeds and 94 water depths.
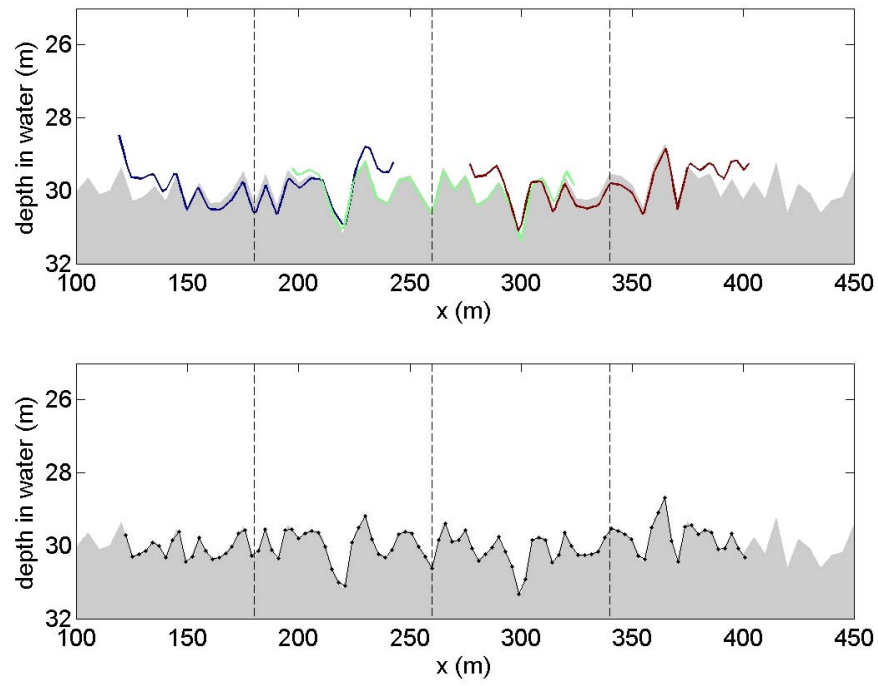

Figure 4. MBES measurement configuration consisting of three parallel tracks. The positions of the MBES are indicated by the vertical black dashed lines. The true bathymetry is indicated by the grey surface. The upper plot presents the estimates for the bathymetry along the three swathes where use is made of erroneous sound speeds. The lower plot presents the estimates for the bathymetry obtained from application of the proposed method.

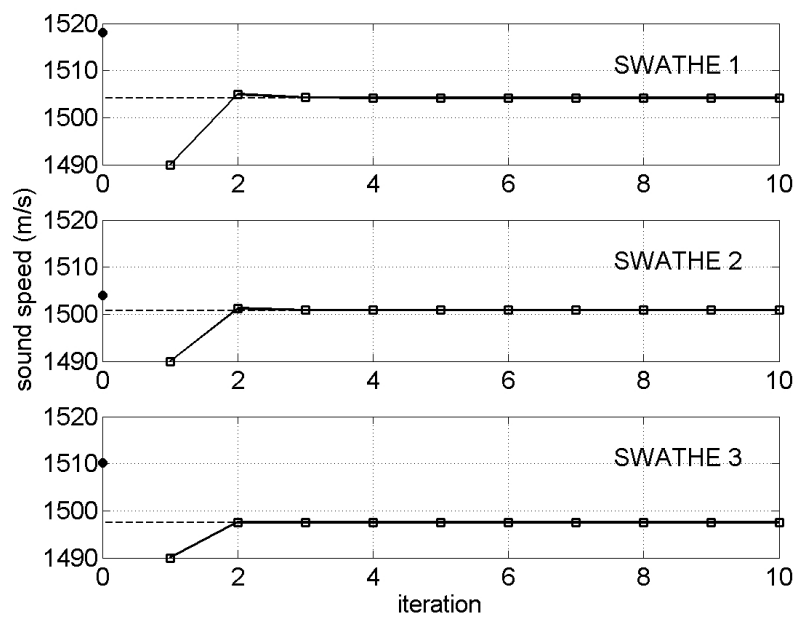

Figure 5. Sound speed estimates as a function of iteration, indicated by the squares. The true sound speed values correspond to the dashed black line. The black circle at iteration zero indicates the "measured" sound speed used for calculating the bathymetry as shown in the upper plot of Fig. 4.

For a further assessment of the method's performance, 100 independent optimizations have been carried out for this configuration with three parallel tracks. Each optimization was carried out for a different bathymetry and a different set of true and measured sound speeds.

Fig. 6 shows the resulting sound speed estimates for these 100 independent optimizations. The black circles indicate the optimized sound speed versus the true sound speeds. The grey circles indicate the measured sound speed versus the true sound speeds. The figure clearly demonstrates the ability of the method to estimate the true sound speeds. The majority of the sound speed estimates almost coincide with the true sound speed value. Due to the computational efficiency of the GaussNewton approach, simulations as presented here can be easily carried out. 


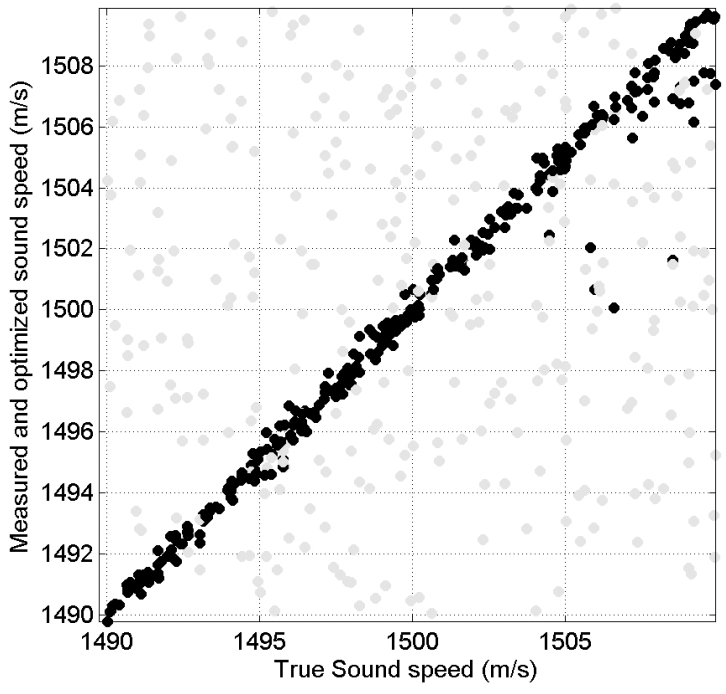

Figure 6. Sound speed estimates obtained from 100 independent optimizations. The situation considered is that of three parallel tracks. The black circles denote the sound speed estimates, plotted versus the true sound speeds. The grey circles indicate the measured sound speed values, also plotted versus the true sound speed.

As a next step, a configuration with 15 parallel tracks is considered. Fig. 7 shows the estimated sound speeds obtained from carrying out 100 independent optimizations. Again it is shown that the proposed method allows for an accurate estimation of the unknown sound speeds.

Fig. 8 and Fig. 9 present the optimized bathymetry and sound speeds for one realization, respectively. From Fig. 8 it is clear that the method is able to determine the true bathymetry, also for the situation with 15 parallel swathes,. From fig. 9 it is seen that this also holds for the sound speeds.

\section{CONCLUSIONS}

In this paper it is demonstrated that by employing the overlap between adjacent MBES swathes, errors in the bathymetry due to erroneous sound speed information, can be eliminated. In principle, this method allows for MBES surveys where no information regarding the prevailing sound speeds is acquired. The only requirement is that sufficient overlap exists between the neighboring swathes. Simulations such as presented in the current contribution allow for a quantification of the required overlap for a certain measurement configuration.

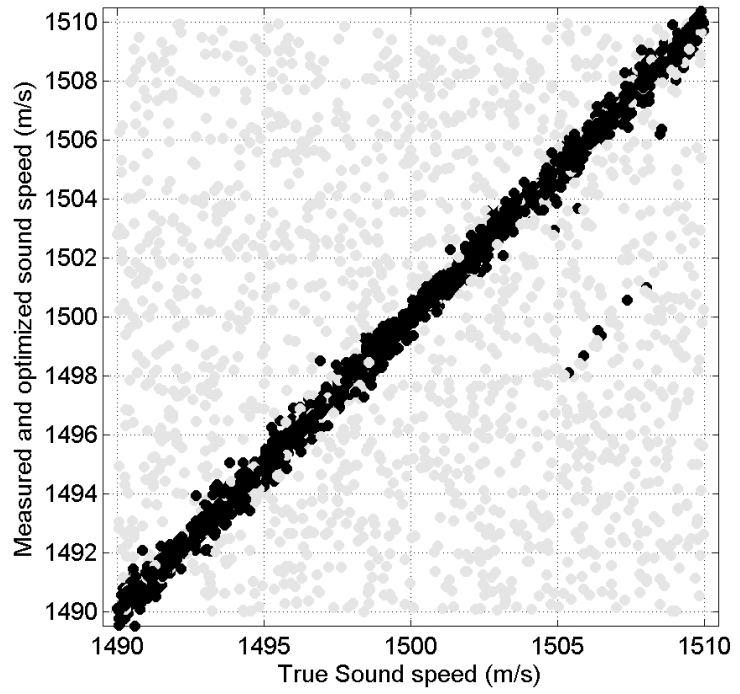

Figure 7. Sound speed estimates obtained from 100 independent optimizations. The situation considered is that of 15 parallel tracks. The black circles denote the sound speed estimates, plotted versus the true sound speeds. The grey circles indicate the measured sound speed values, also plotted versus the true sound speed.

\section{ACKNOWLEDGMENT}

We would like to thank Ben Dierikx and the Dutch Directorate-General for Public Works and Water Management for useful discussions and for providing information on typical MBES survey geometries. This work is financially supported by the Dutch Directorate-General for Public Works and Water Management, the Netherlands Geodetic Commission, and the Technical University of Delft.

\section{REFERENCES}

[1] J. D. Beaudoin, J. E. Hughes Clarke, J. E. Bartlett, “Application of surface sound speed measurements in postprocessing for multi-sector multibeam echosounders", International Hydrographic Review 5 (3), 26-31 (2004)

[2] D. S. Cartwright, J. E. Hughes Clarke, "Multibeam surveys of the frazer river delta, coping with an extreme refraction environment", In Proceedings of the Canadian Hydrographic Conference, Montreal, Canada (2002)

[3] D. F. Dinn, B. D. Loncarevic, G. Costello, "The effect of sound velocity errors on multi-beam sonar depth accuracy", In OCEANS '95. MTS/IEEE. Challenges of Our Changing Global Environment. Conference Proceeding, San Diego, CA, USA (1995)

[4] M. Snellen, J.P. van den Ameele, R. Biersteker, D. G. Simons, "Improving multi-beam echo sounder depth measurements", In Proceedings of the 15th International Congress of the International Federation of Hydrographic Societies. Evolutions in Hydrography, Hydro06, Antwerpen, Belgium, 6-9 november 2006, Special Publication, 55, 97-101 (2006)

[5] J. P. van den Ameele, "Improving MBES measurements", Hydro International 11(2), 27-29 (2007) 

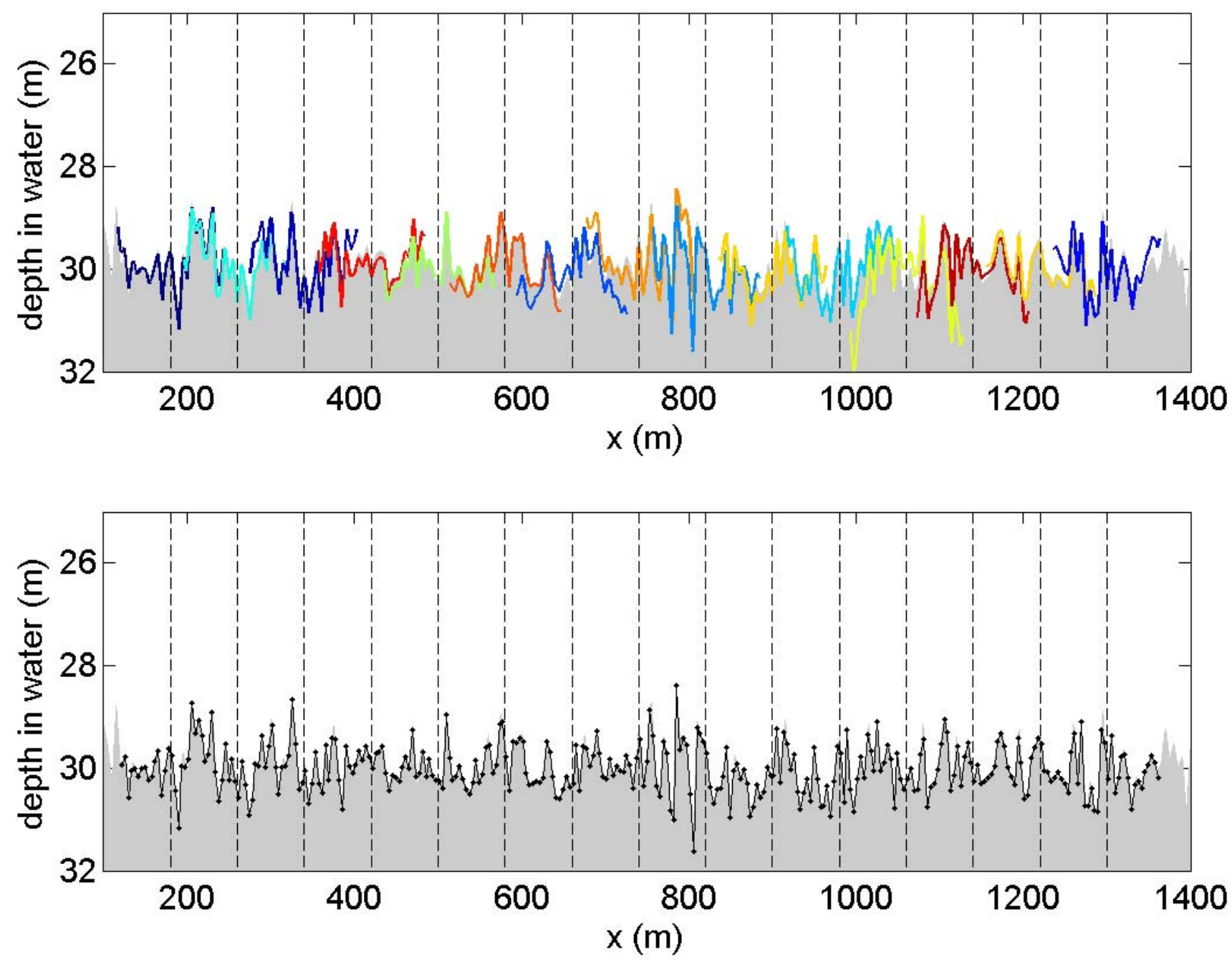

Figure 8. MBES measurement configuration consisting of 15 parallel tracks. The positions of the MBES are indicated by the vertical black dashed lines. The true bathymetry is indicated by the grey surface. The upper plot presents the estimates for the bathymetry along the three swathes where use is made of erroneous sound speeds. The lower plot presents the estimates for the bathymetry obtained from application of the proposed method. 

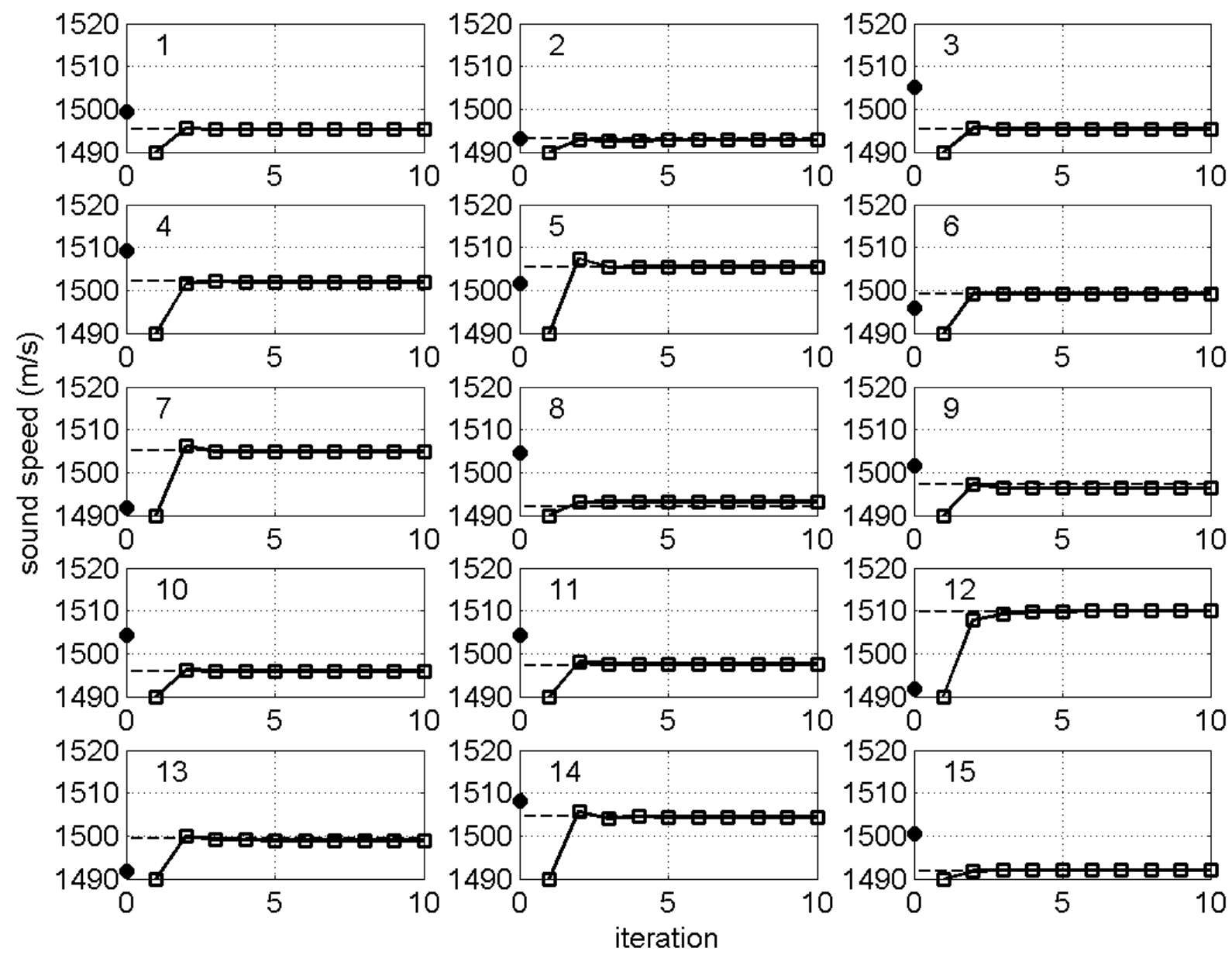

Figure 9. Sound speed estimates as a function of iteration, indicated by the squares. Swathes are indicated by the numbers in the subplots. The true sound speed values correspond to the dashed black line. The black circle at iteration zero indicates the "measured" sound speed used for calculating the bathymetry as shown in the upper plot of Fig. 8. 